\title{
Role of Male Partners in 100 Infertile Couples
}

\author{
Ara $\mathrm{MJ}^{1}$, Hussain $\mathrm{SMB}^{2}$, Rashid $\mathrm{MU}^{3}$
}

\begin{abstract}
Introduction: Approximately $15 \%$ of couples are infertile. Male infertility plays an important role in about half of these couples. This study has been done to determine whether sperm morphology and motility do really affect sperm count or spermatogenesis and at the same time causes a problem with fertility.
\end{abstract}

Objectives: The objective of this study was to evaluate a male's inability to cause pregnancy in a fertile female.

Materials and Methods: This is a prospective study of 100 diagnosed infertile and subfertile couples in the age group of $23-58$ yrs old male. They were selected from 01 March 2013 to 28 Feb 2014 undergoing treatment for infertility in Dhaka $\mathrm{CMH}$. On the basis of sperm count the cases were grouped as follows: Normozoospermia, oligozoospermia \& Azoospermia. Semen analysis was done in all the cases and the results were described with arithmetic mean and standard deviation.

Results: Normozoospermia: Mean and SD of sperm count, sperm abnormality, sperm motility are $65.98 \pm$ $5.05,24.44 \pm 1.57$ and $45.5 \pm 2.94$ respectively; Oligozoospermia: Mean and SD of sperm count, sperm abnormality, sperm motility are $7.74 \pm 1.23$, $41.1 \pm 3.78,14.54 \pm 2.77$ respectively; Azoospermia: Mean and SD of sperm count, sperm abnormality, sperm motility are absent.

Conclusion: Here data statistically showed person with normozoospermia having good sperm morphology and motility than oligozoospermia and azoospermic subjects thereby causing male infertility which is responsible for hindrance in achieving pregnancy clinically.
Key-words: Normozoospermia, Oligozoospermia, Azoospermia, infertile couple, subfertile couple.

\section{Introduction}

Infertility is a significant medical problem that affects many couples. Infertility is a disease of the reproductive system defined by the failure to achieve a clinical pregnancy after 12 months or more of regular unprotected sexual intercourse ${ }^{1}$. Male infertility refers to a male's inability to cause pregnancy in a fertile female ${ }^{2}$. Approximately $15 \%$ of couples are infertile ${ }^{1,2,3}$. Male infertility plays an important role in about half of these couples ${ }^{1}$. Male infertility is commonly due to deficiencies in the semen and semen analysis is a vital part of diagnosing male infertility ${ }^{2,4}$. The volume of the semen sample, approximate number of total sperm cells, sperm motility/forward progression and percent of sperm with normal morphology are measured. This is the most common type of fertility testing ${ }^{2,4}$. Semen deficiencies are often labeled as follows: Oligospermia or oligozoospermia- decereased number of total sperm cells in semen; Azoospermiaabsence of sperm cells in semen; Teratospermiaincrease in sperm with abnormal morphology and Asthenozoospermia- reduced sperm motility.

A normal semen analysis often labeled as follows: Normozoospermia-Normal count of sperm cells in semen, (20-150 million/ml); Spermatogenesis-The process of male gamete formation including formation of a spermatocyte from a spermatogonium, meiotic division of the spermatocyte and transformation of the four resulting spermatids into into spermatozoa; Sperm morphology- The form and structure of a sperm; Sperm motility- Forward progression of a sperm. For many decades, we

1. Lt Col Mah Jabeen Ara, MBBS, DCP (Clin path), MCPS (Clin path), FCPS (Chem path), CMH, Jalalabad;

2. Brig Gen Sk Md Bahar Hussain (Retd), MBBS, FCPS (Medicine), FRCP, FACP (Retd), United Hospital;

3. Maj Mamun ur Rashid, MBBS, MCPS, FCPS (Radiology and imaging), CMH, Jalalabad. 
focused mainly on sperm count and motility when doing a semen analysis. For the past 25 years with the advent of In Vitro Fertilization (IVF), the importance of sperm morphology (shape) is being realized. Sperm morphology reflects the quality of sperm production (Spermatogenesis). Abnormal morphology (teratospermia) is associated with male factor infertility with reduced fertilization and pregnancy rates. This study has been done to determine whether sperm morphology and motility do really affect sperm count or spermatogenesis and at the same time causes a problem with fertility.

\section{Materials and Methods}

Study Design: This is a prospective study carried out with infertile and subfertile couples treated in Dhaka CMH in the period from 01 March 2013 to 28 February 2014.

Patients: Semen specimens were obtained from 100 consecutive male patients between 23 and 58 years of age. These patients attended clinical pathology laboratory, Armed Forces Institute of Pathology (AFIP) for fertility evaluation. All subjects or patients were asked to produce a first semen sample after a sexual abstinence of 3 days, as because increase in abstinence with individual days significantly affects semen volume, sperm count, sperm motility and vitality. In particular, sperm motility (percent of sperm movement) peaked after one day of abstinence in men with infertility problems. A new study shows that sperm from men with low sperm counts reached their peak condition after one day of abstinence ${ }^{5}$.

Data Collection: Data were collected through interview of couples who did not have child for last one year or longer even though they have had frequent unprotected sexual intercourse or couples had a child but now they are unable to procreate due to some unknown reason using structured questionnaire. Sample size was 150 (100 infertile \& subfertile couple and 50 control subjects).

Criteria: According to WHO criteria; Normozoospermia is defined as an ejaculate with sperm concentration of $>20 \times 10^{6}$ spermatozoa $/ \mathrm{ml}$ progressive sperm motility of $>50 \%$ and $>30 \%$ of morphologically normal spermatozoa ${ }^{6}$.
Assessment of Sperm motility and concentration: The evaluation of sperm motility and concentration was performed by using light microscope. After liquefaction, $10 \mu \mathrm{l}$ of semen was pipetted onto a glass slide and covered with a cover slip (size $18 \times 18 \mathrm{~mm}$ ). The analysis was performed at room temperature at final magnification of 100x. The motility was expressed as the percentage of motile spermatozoa: Spermatozoa with linear and progressive motility (linear velocity $>22 \mu \mathrm{m} / \mathrm{s}$ ); Spermatozoa with slow linear or nonlinear motility (linear velocity $<22 \mu \mathrm{m} / \mathrm{s}$ and velocity $>5 \mu \mathrm{m} / \mathrm{s}$ ); Sluggish, and Immotile spermatozoa. Sperm morphology assessment was done by WHO criteria, $^{2,6,7}$ : Following liquefaction, $10 \mu \mathrm{l}$ of semen was spread onto a glass slide and allowed to air dry at room temperature. The smears were then stained with giemsa stain and sperm morphology was assessed according to WHO criteria.

Characteristics of normal sperm: According to WHO criteria, a normal sperm has: a smooth, oval

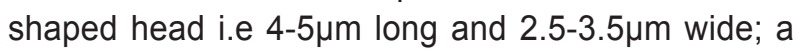
well-defined acrosome that covers $40 \%-70 \%$ of the head; The midpiece is thin, less than $1 \mu \mathrm{m}$ wide; Cytoplasmic droplets, if present, should not be larger than half of the head width; The tail is thin, uniform, uncoiled and about $45 \mu \mathrm{m}$ long. According to this classification system, all borderline forms are considered as abnormal.

Statistical analysis: The results were described with arithmetic mean and standard deviation. Control included $(n=50)$.

\section{Results}

Table-I: Shows ages of patients. These patients are classified into three groups like Normozoospermia, Azoospermia, Oligozoospermia.

\begin{tabular}{|l|c|c|c|}
\hline Patients & $\mathbf{N}(\mathbf{n}=59)$ & $\mathbf{A ( n = 2 2 )}$ & $\mathbf{O ( n = 1 9 )}$ \\
\hline Age (Years) & $33.5 \pm 5.8$ & $31 \pm 6.9$ & $39 \pm 6.6$ \\
\hline
\end{tabular}

$\mathrm{N}=$ Normozoospermia, $\mathrm{A}=$ Azoospermia,

$\mathrm{O}=$ Oligozoospermia, $\mathrm{n}=$ Number of subjects.

Table-II: Shows different parameters of patient's semen.

\begin{tabular}{|l|c|c|c|}
\hline Patients & $\mathbf{N}$ (n=59) & $\mathbf{A ~ ( n = 2 2 ) ~}$ & $\mathbf{0}$ (n=19) \\
\hline Volume (m) & $3.36 \pm .16$ & $2.36 \pm .24$ & $2.5 \pm .17$ \\
\hline Sexual abstinence(days) & $4 \pm 1$ & $4 \pm 2$ & $4 \pm 1$ \\
\hline Semen pH & $7.90 \pm .21$ & $7.92 \pm .24$ & $8.11 \pm .38$ \\
\hline
\end{tabular}

$\mathrm{N}=$ Normozoospermia, $\mathrm{A}=\mathrm{Azoospermia}$,

$\mathrm{O}=$ Oligozoospermia, $\mathrm{n}=$ Number of subjects. 
Table-III: Shows concentration of sperms of patients.

\begin{tabular}{|l|c|c|c|}
\hline Patients & $\mathrm{N}(\mathrm{n}=\mathbf{5 9})$ & $\mathrm{A}(\mathrm{n}=\mathbf{2 2})$ & $\mathbf{0}(\mathrm{n}=\mathbf{1 9})$ \\
\hline Sperm count $\left(\mathrm{x} 10^{6} / \mathrm{ml}\right)$ & $65.98 \pm 5.05$ & Nil & $7.74 \pm 1.23$ \\
\hline
\end{tabular}

$\mathrm{N}=$ Normozoospermia, $\mathrm{A}=\mathrm{Azoospermia}$,

$\mathrm{O}=$ Oligozoospermia, $\mathrm{n}=$ Number of subjects.

Table-IV: Shows correlation of morphology and motility between Normozoospermic \& Oligozoospermic subjects.

\begin{tabular}{|l|c|c|c|}
\hline Patients & $\mathbf{N}(\mathbf{n}=\mathbf{5 9})$ & $\mathbf{A}(\mathbf{n}=\mathbf{2 2})$ & $\mathbf{0}(\mathbf{n}=\mathbf{1 9})$ \\
\hline Sperm abnormality & $24.44 \pm 1.57$ & Nil & $41.10 \pm 3.78$ \\
\hline Sperm motility & $45.5 \pm 2.94$ & Nil & $14.54 \pm 2.77$ \\
\hline
\end{tabular}

$\mathrm{N}=$ Normozoospermia, $\mathrm{A}=$ Azoospermia,

$\mathrm{O}=$ Oligozoospermia, $\mathrm{n}=$ number of subjects

\section{Discussion}

Assessment of sperm morphology as a component of semen analysis is one of the most important steps in the evaluation of male partner in infertile couples. In some cases, morphology is used to decide whether a couple should use IVF to attempt a pregnancy ${ }^{8,9}$. Having higher amounts of abnormally shaped sperm has been associated with infertility in some studies ${ }^{10}$. Usually, a higher number of abnormal shaped sperm are associated with other irregularities of the semen such as low sperm count or motility and at the same time causes a problem with fertility ${ }^{11}$.

In this study, it is clearly visible when sperm morphology is $<30 \%$ abnormal the count increases i.e becoming normozoospermia and sperm count decreases when sperm morphology is $>30 \%$ abnormal. The findings of this study matches with the study led by Eustache F, Auger $\mathrm{J}^{12}$. This study is also supported by the study of Menkveld R, Stander $\mathrm{FS}^{7}$. Likewise this study also shows that motility of sperm when $>50 \%$ the count of sperm is normal on the contrary oligozoospermic patient have less sperm motility which is similar to the study done by Guzick DS, Overstreet $\mathrm{JW}^{11}$. The study of Eustache $\mathrm{F}$, Auger $\mathrm{J}$ also supports this study ${ }^{12}$. Sperm motility is one of the important factors in male fertility ${ }^{11}$.

If the sperm motility is low it may hinder one's ability to conceive. Sperm with healthy motility move progressively forward, not sluggishly and not traveling in circles. Specialists have four motility grades they give to sperm, from an 'A' for the straightest, fastest swimmers, to a ' $D$ ' for sperm that fail to move at all, ${ }^{2,7}$. When a sample shows that fewer than $32 \%$ of sperm do not swim forward progressively, the motility of that sample is considered low $^{11}$. In this study oligozoospermic patients have low sperm motility i.e $14.54 \%$. This study matches with the study of 'WHO' after studying semen from 4000 fertile men $^{13}$. In some cases, morphology is used to decide whether a couple should use in vitro fertilization (IVF) to attempt a pregnancy ${ }^{8}$. Men with abnormally shaped sperm tend to have more trouble causing a pregnancy and at the same time cause a problem with fertility ${ }^{14}$. This study shows patients having $>50 \%$ abnormal shaped sperm are suffering from subfertility which conforms to the studies led by Kruger TF, Acosta AA, Simmons KF et al ${ }^{9,15,16}$.

\section{Conclusion}

The study showed person with oligozoospermia and azoospermia causing male infertility. Sperm count can be increased by taking antioxidant vitamins, weight loss and other life style changes.

\section{References}

1. Gurunath S, Pandian Z, Anderson RA et al. Defining infertility-a systematic review of prevalence studies. Human Reproduction update 1999; 17(5):575-88.

2. Rowe PJ, Comhaire FH, Hargreave TB, Mahmoud AMA (2000). WHO manual for the standardized investigation, diagnosis and management of the infertile male. Cambridge (England): Published on behalf of the WHO by Cambridge University press; p5-16.

3. Makar RS, Toth TL. The evaluation of infertility. Am J Clin pathol(2002); 117:95-103

4. Cooper TG, Noonan E, Von Eck ardstein S, et al. World Health Organization reference values for human semen characteristics. Hum. Reprod. Update 2000;16:231-45.

5. Levitas E. Fertility and Sterility. Reprod Biol Endocrinol June 2005,83:1680-6.

6. Barroso G, Mercan R, Ozgur $\mathrm{K}$ et al. Intra and inter-laboratory variability in the assessment of sperm morphology by strict criteria: impact of semen preparation, staining techniques and manual versus computerized analysis. Hum Reprod 1999; 14:2036-40.

7. Menkveld R, Stander FS, Kotze TJ et al. The evaluation of morphological characteristics of human spermatozoa according to stricter criteria. Hum. Reprod. 1990;5:586.

8. Sukcharoen N, Keith J, Irvine DS et al. Prediction of the invitro fertilization (IVF) potential of human spermatozoa using sperm function tests, the effect of the delay between testing and IVF. Hum Reprod 1996;11:1030. 
9. Kruger TF, Acosta AA, Simmons KF et al. Predictive value of abnormal sperm morphology in invitro fertilization. Fertil steril 1988; 49:112-7.

10. Glatstein IZ, Harlow BL, Hornstein MD. Practice patterns among reproductive endocrinologists. Further aspects of the infertility evaluation. Fertil Steril 1998; 70:263.

11. Guzick DS, Overstreet JW, Factor-LitvakP, et al. Sperm morphology, motility and conc. fertile \& infertile men. N Engl J Med 2001; 345:1388-93.

12. Eustache F, Auger J. Inter indl Variability in the morphological assessment of human sperm: effect of the level of experience and the use of standard methods. Hum Reprod 2003;18:1018-22.
13. World Health Organization. WHO Laboratory Manual for the Examination of Human semen and semen-cervical Mucus Interaction. 4th ed. Cambridge University Press; 1999.

14. Robbins WA, Elashoff DA, Xun L et al. Effect of lifestyle exposures on sperm aneuploidy. Cytogenetic and Genome Research III,(2005),(3-4):371-7.

15. Gunalp S, Onculoglu C, Gurgan T et al: A study of semen parameters with emphasis on sperm morphology in a fertile population: an attempt to develop clinical thresholdes. Hum Reprod 2001; 16:110.

16. Brooks AK. How reliable are results from the semen analysis? Fertil steril 2004; 82:41-4. 